\title{
Evaluation of the clinical performance of ultrahigh molecular weight polyethylene fiber cable using a dog osteosynthesis model
}

\author{
Kenichi Oe ${ }^{\text {a }}$, Seiya Jingushi ${ }^{\mathrm{b}, *}$, Hirokazu Iida $^{\mathrm{a}}$ and Naohide Tomita ${ }^{\mathrm{c}}$ \\ ${ }^{a}$ Department of Orthopaedic Surgery, Kansai Medical University, Osaka, Japan \\ ${ }^{\mathrm{b}}$ Department of Orthopaedic Surgery, Kyushu Rosai Hospital, Kitakyushu, Japan \\ ${ }^{\mathrm{c}}$ Bioengineering Laboratory, Graduate School of Engineering, Kyoto University, Kyoto, Japan
}

Received 18 January 2012

Revised 4 February 2013

\begin{abstract}
The purpose of this study was to assess the removability and biological reactivity of an ultrahigh molecular weight polyethylene (UHMWPE) fiber cable as a new biomaterial for osteosynthesis. We used a pull-out test and an implantation test to analyze the performance of the UHMWPE fiber cable using a dog model, and compared its characteristics with those of a wire cable and a soft wire. In the pull-out test, the UHMWPE fiber cable was as easy to remove as the soft wire, and both the UHMWPE fiber cable and the soft wire were significantly easier to remove than the wire cable. The fixation capability and the biological reactivity of the UHMWPE fiber cable were examined in an osteosynthesis model of the dog greater trochanter, and were compared with those of the soft wire. The bone union rate, assessed radiographically, was very similar when using the UHMWPE fiber cable and the soft wire. However, in the soft wire group, both the surface of the greater trochanter under the fixation material and the penetration area around the fixation material showed an increased tendency toward a biological reaction, including inflammation and granulation tissue formation, as compared to the UHMWPE fiber cable group. The UHMWPE fiber cable was as easily removed from the bone tissue as the soft wire, and was easier to remove than the wire cable. Additionally, the UHMWPE fiber cable caused reduced biological reactivity with the surrounding tissue, as compared with the soft wire. In conclusion, the UHMWPE fiber cable appeared to be a suitable fixation material for osteosynthesis.
\end{abstract}

Keywords: Ultrahigh molecular weight polyethylene fiber cable, biomaterials, osteosynthesis, greater trochanter osteotomy

\section{Introduction}

An encircling metal fixation device, such as a steel wire or titanium cable, has long been used as an orthopedic reconstructive appliance. Such implants have been used in the internal fixation of fractures and the re-attachment of the osteotomized greater trochanter in total hip arthroplasty (THA), revision THA, or osteotomy. However, even these implants have some disadvantages. Frequent postoperative problems occurring after re-attachment of the greater trochanter in THA include breakage of fixation

\footnotetext{
*Address for correspondence: Seiya Jingushi, Department of Orthopaedic Surgery, Kyushu Rosai Hospital, 1-1 Sone Kita-machi, Kokura Minami-ku, Kitakyushu 800-0296, Japan. Tel.: +81 93471 1121; Fax: +81 93473 5903; E-mail: jingushi.orth@kyushuh.rofuku.go.jp.
} 


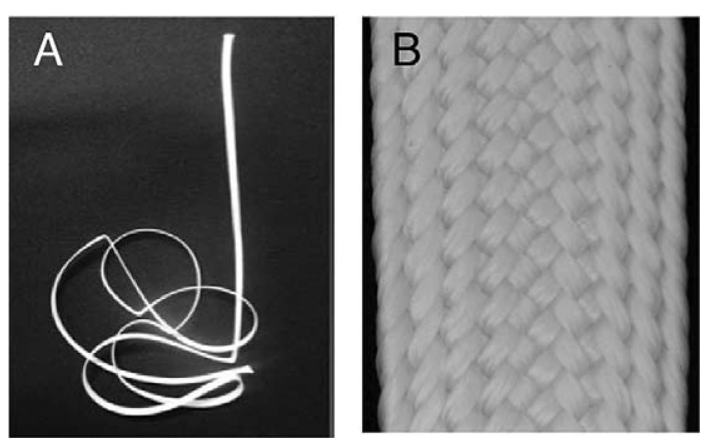

Fig. 1. Photograph of the UHMWPE fiber cable. (A) Gross appearance. (B) Close-up appearance (original magnification $\times 30$ ). The cable is a soft flexible material in the form of a tape.

materials, trochanteric nonunion, and bursitis. The breakage rate has been reported to be $0.4-28 \%$ for steel wire [1-5] and 3.1-43\% for wire cable [6-9]. Wire failure because of low cycle fatigue remains the most common problem. Cable fraying and fragmentation have been reported to develop in 50\% of patients, and the use of cables has limited effectiveness in reducing the incidence of trochanteric nonunion [6,7]. Furthermore, a $1 \%$ notch has been reported to be enough to reduce the fatigue resistance of the wire by $63 \%$ [10]. Trochanteric nonunion rates of $0.4-21 \%$ for steel wire [1-5] and $1.5-38 \%$ for wire cable have been reported [6-9]. Trochanteric nonunion may lead to pain, limp, and postoperative dislocation. It is also easy to understand how these metal implants could cause local bursitis. The need for greater strength to resist both fatigue and static tensile forces, as well as reduce abrasiveness, has prompted the development of new materials for use as an orthopedic reconstructive appliance.

An ultrahigh molecular weight polyethylene (UHMWPE) fiber cable is a soft flexible material created in the form of a tape (Fig. 1). It is used to bind a metal rod to the bone in spinal surgeries due to its strong fixation ability. In spinal fusion surgeries, Dickman et al. reported that UHMWPE fiber cables are superior in static ultimate tensile strength, fatigue strength, conformance, and abrasion as compared with titanium cable, steel cable, or steel wire [11]. Furthermore, Murakami et al. reported that UHMWPE fiber cables perform better than steel wire or titanium cable in the laminar cut-through test and the biomechanical stiffness test [12]. That is, while UHMWPE fiber cables are comparable to metal implants with regard to tensile strength and tensile stiffness, this material also results in fewer failures due to problems such as breakage or abrasion. Additionally, UHMWPE fiber cables is made as a soft, wide, flat tape and thus, is useful for binding as well as causing less local stress with little possibility of cutting into bones. This may ameliorate the 'cheese wire effect' that can occur during fixation of fragile bones in senile osteoporotic patients. Therefore, we hypothesized that a UHMWPE fiber cable would be an excellent material for use in osteosynthesis as well as for binding a metal rod to the bone in spinal surgeries. However, the area of contact with bone tissue would be larger than in spinal surgeries, and the UHMWPE fiber cable could run through the bone when used for osteosynthesis. In general, fixation materials for osteosynthesis should be removable after bone union is achieved. In addition, the biological reactivity of any implant material with bone should be assessed before clinical use. The purpose of this study was to evaluate the removability and the biological reactivity of a UHMWPE fiber cable when used for osteosynthesis. We used a pull-out test and an implantation test to assess the use of a UHMWPE fiber cable for osteosynthesis in a large animal model. 


\section{Materials and methods}

\subsection{Biomaterials}

A UHMWPE fiber cable (NESPLON Cable System, Alfresa Pharma Co., Osaka, Japan) $3.0 \mathrm{~mm}$ in width was used. Each UHMWPE fiber cable comprised 8 yarns of fiber, each consisting of 120 fibers, braided to form a cable approximately $1.0 \mathrm{~mm}$ in diameter (Fig. 1). Wire cable (Ti-6Al-4V Alloy Wiring System, Nakashima Propeller Co., Ltd, Okayama, Japan) $1.2 \mathrm{~mm}$ in diameter and soft wire (stainless steel, B. Braun Aesculap Japan Co., Ltd, Tokyo, Japan) $0.8 \mathrm{~mm}$ in diameter were used as control materials.

In a preliminary study, the physical properties of each material were evaluated by tensile tests for the cables and wire using Tensilon RTC-1250A (A\&D Co., Ltd, Tokyo, Japan), where the inner edge length was $254 \mathrm{~mm}$ and the cross-head velocity was $254 \mathrm{~mm} / \mathrm{min}$. The withstand load of the UHMWPE fiber cable, soft wire and wire cable were $626 \pm 26,710 \pm 4$ and $851 \pm 68 \mathrm{~N}$, respectively. Practical strength of the UHMWPE fiber cable, soft wire, and wire cable were evaluated by knot strength, twist splicing strength, and crimping strength, respectively [11]. These tests were performed for looped samples loaded by hooks measuring $20 \mathrm{~mm}$ in diameter with an inner edge length of $254 \mathrm{~mm}$ and at a cross-head velocity of $126 \mathrm{~mm} / \mathrm{min}$ using an Instron 5582 Universal Testing Machine (Instron Japan Co., Ltd, Kanagawa, Japan). The UHMWPE fiber cable was looped by tying with double loop-sliding knot technique using a special gun-type apparatus (Alfresa Pharma Co.). The soft wire was tied by twisting over 4 circuits using KY220S (B. Braun Aesculap Japan Co., Ltd), and the wire cable was crimped with a special loute wire tightening instrument (Cable Tensioner, Nakashima Propeller Co., Ltd) which cuts the wire cleanly without leaving any notches (Crimper, Nakashima Propeller Co., Ltd). The results for the UHMWPE fiber cable, soft wire, and wire cable were $1301 \pm 68,672 \pm 21$ and $972 \pm 95 \mathrm{~N}$, respectively. The fatigue strength test was performed with $5 \times 10^{6}$ cycles of $300 \mathrm{~N}$. The results for the UHMWPE fiber cable, soft wire, and wire cable were over $5 \times 10^{6}, 4.04 \times 10^{4}$ and $3.31 \times 10^{3}$ cycles, respectively.

\subsection{Animals}

Ten- or eleven-month-old female beagles (TOYO Beagles), weighing 7.6-10.7 kg, were purchased from KITAYAMA LABES Co. Ltd (Nagano, Japan). The beagles were maintained in our animal facility under specific pathogen-free conditions. All surgical procedures and the postoperative care of the animals were carried out at the Bozo Research Center Inc., Tokyo, Japan, in accordance with the guidelines of the National Institutes of Health for the care and use of primates. The study protocol was reviewed and approved by the Bozo Research Center Animal Experimentation Committee.

\subsection{Experiment 1: Pull-out test}

The dogs $(N=6)$ were pretreated with atropine sulfate (Tanabe Seiyaku Co., Ltd, Osaka, Japan) and were anesthetized intravenously with pentobarbital sodium $(30 \mathrm{mg} / \mathrm{kg}$, Tokyo Chemical Industry Co., Ltd, Tokyo, Japan). The anterolateral part of the left femur was exposed, and 3 holes were prepared in the shaft of the femur at approximately $10 \mathrm{~mm}$ intervals using a drill $2.0 \mathrm{~mm}$ in diameter. An UHMWPE fiber cable $(N=6)$, soft wire $(N=6)$ and wire cable $(N=6)$ were then implanted in each femur by passing them through the 3 different holes and then encircling the femur several times. Finally, the UHMWPE fiber cable was tightened with a special gun-type apparatus (Alfresa Pharma Co.). The soft wire was tied by twisting over 4 circuits using KY220S (B. Braun Aesculap Japan Co., Ltd), and the wire cable 
was crimped with a special loute wire tightening instrument (Cable Tensioner, Nakashima Propeller Co., Ltd), which cuts the wire cleanly without leaving any notches (Crimper, Nakashima Propeller Co., Ltd). The subcutaneous tissue was sutured with 3-0 vicryl (Johnson and Johnson K.K., Tokyo, Japan) and the skin was sutured with 2-0 nylon (Johnson and Johnson K.K.). Streptomycin sulfate (MEIJI SEIKA KAISHA, Ltd, Tokyo, Japan) and benzylpenicillin potassium (MEIJI SEIKA KAISHA) were administered locally to the operative field during surgery, and azithromycin hydrate (Pfizer Japan Inc., Tokyo, Japan) was administered orally for 2 days after surgery.

Tests were performed 6 months after implantation. After necropsy, the femur was removed en bloc, cut across using a circular saw, and the bone marrow in the canal was removed. Then, the surrounding materials were removed. To remove the materials in the canal, the UHMWPE fiber cable was cut off with scissors, the soft wire was cut off using a $2.54 \mathrm{~mm}$ diameter router at $3 \times 10^{4} \mathrm{rpm}$, and the wire cable was cut off with a nipper. When the wire cable was cut, it was allowed to straighten gradually to detach gently from the soft tissues without damaging them.

Finally, the binding part was cut off. During all procedures, normal saline was instilled appropriately to prevent dryness of the bone. Using Tensilon RTC-1250A (A\&D Co., Ltd), the bone was fixed at the bottom and the test material was fixed at the top $4 \mathrm{~mm}$ away from the bone hole (Fig. 2, right panel). A pull-out test was then performed at $10 \mathrm{~mm} / \mathrm{min}$.

\subsection{Experiment 2: Implantation test of fixation for re-attachment of the greater trochanter}

An UHMWPE fiber cable $(N=12) 3.0 \mathrm{~mm}$ in width was used as the test material, while a soft wire $(N=12)$ of $0.8 \mathrm{~mm}$ in diameter was used as the control material. The wire cable was not included in the pilot study because the binding portion of the wire cable designed for human use was too big for use in dogs. Until sacrifice of the animals, no problems with fixation were observed in the UHMWPE fiber cable group. In the soft wire group, wire breakage was observed in 1 animal, and early detachment of the greater trochanter fragment was observed in 2 animals. Those animals were excluded from this experiment, and additional animals were operated on later.
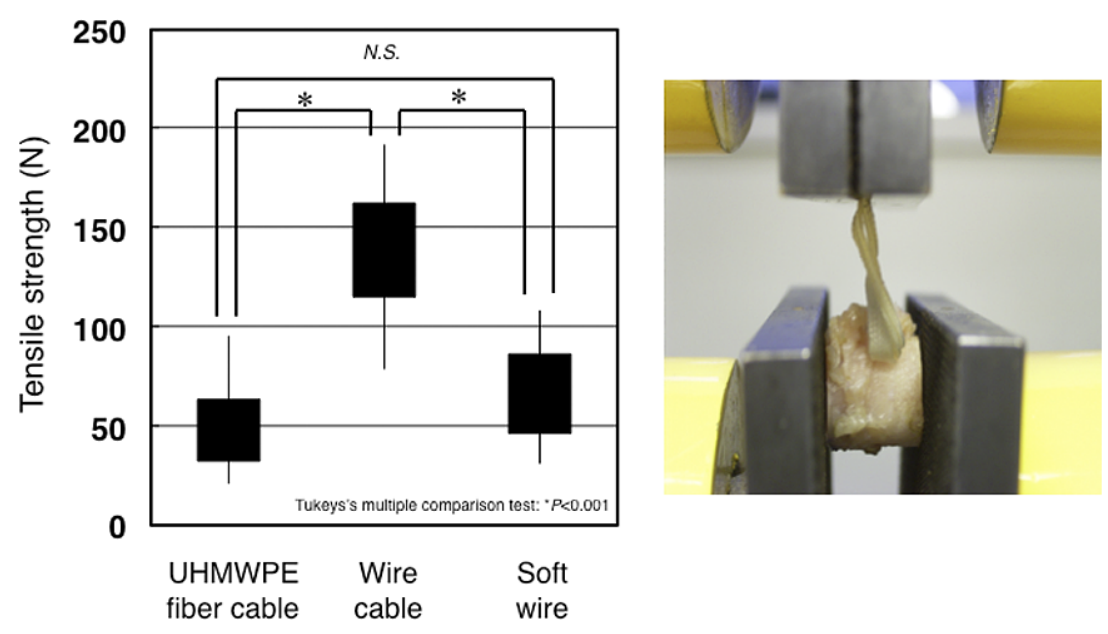

Fig. 2. The pull-out strength at the insertion site of various fixation materials. The right photograph shows how the test was carried out in the UHMWPE fiber cable sample. The ultimate failure load using a UHMWPE fiber cable, wire cable, or soft wire is shown in the left graph. The results are expressed as the mean \pm standard deviation. (Colors are visible in the online version of the article; http://dx.doi.org/10.3233/BME-130757.) 
The dogs $(N=24)$ were pretreated with atropine sulfate and anesthetized intravenously with pentobarbital sodium $(30 \mathrm{mg} / \mathrm{kg})$. The greater trochanter of the left femur was cut, leaving the abductor muscle intact. Holes were made in the bone fragment of the greater trochanter and the femur using a drill $2.0 \mathrm{~mm}$ in diameter. The bone fragment was returned to the original position and bound in place using an UHMWPE fiber cable or a soft wire, which was passed through the holes. The UHMWPE fiber cable was tightened using a double loop-sliding knot technique with a special gun-type apparatus (Alfresa Pharma Co.). The soft wire was tied by twisting over 4 circuits using KY220S (B. Braun Aesculap Japan Co., Ltd). The subcutaneous tissue and skin were sutured with a surgical thread of 3-0 vicryl and 2-0 nylon, respectively. Streptomycin sulfate and benzylpenicillin potassium were administered locally in the operative field during the operation, and azithromycin hydrate was administered orally for 2 days after surgery.

Radiological evaluations were carried out at 1, 3 and 6 months after implantation. Each dog was pretreated with atropine sulfate and anesthetized intravenously with pentobarbital sodium $(30 \mathrm{mg} / \mathrm{kg})$. Soft X-ray photographs (VPX-40A, TOSHIBA MEDICAL SUPPLY Co., Ltd, Tochigi, Japan) were taken of the hip joint from 4 directions for each animal under the following conditions: $3.2 \mathrm{mAs}, 46-$ $66 \mathrm{kV}$, and 0.01-0.2 s of exposure. Bone union was determined to have occurred if the osteotomy line had disappeared in views from 2 or more directions. The dogs were sacrificed at 1, 3 or 6 months after implantation.

The proximal femur including the greater trochanter was fixed for 3 days in 10\% formalin (FALMA Inc., Tokyo, Japan). Bone samples were then decalcified using EDTA (Wako Pure Chemical Industries Ltd, Osaka, Japan) for 7 days. The soft wire was removed at the end of the decalcification process, while specimens attached with UHMWPE fiber cable were prepared together with the cable. Histological specimens from the areas of the greater trochanter surface, bone penetration, and osteosynthesis were prepared. The sections were stained with hematoxylin and eosin (H-E). The presence of any inflammatory reaction, granulation tissue formation, or fibrosis, or the existence of foreign body giant cells around the fixation material was assessed in the stained sections (modified Naughton's inflammatory response score [13]). An independent investigator at the Bozo Center graded each histological characteristic from 0 to 3 (0: none, 1: occasional, 2: slight, 3: moderate). In addition, bone union was evaluated histologically.

\subsection{Statistical analysis}

The data in Experiment 1 were analyzed by Dunnett's multiple comparison test. The data in Experiment 2 were first analyzed by the $F$-test for homogeneity of variance and then subjected to the Student's $t$-test in cases of homogeneous data and the Aspin-Welch $t$-test in cases of heterogeneous data. Values with a critical level of less than 0.05 were regarded as having statistically significant differences. All data were analyzed using the Bozo Research Center Toxicology System (Bozo Research Center Inc., Tokyo, Japan) and EXAS Ver. 7.1.6 (Arm Systex Co. Ltd, Osaka, Japan). The results are shown as means \pm standard deviations.

\section{Results}

\subsection{Experiment 1}

In the pull-out test, the mean pulling force in the samples of UHMWPE fiber cable, wire cable, and soft wire were $50.2 \pm 26.0,137.7 \pm 32.7$ and $68.4 \pm 26.3 \mathrm{~N}$, respectively (Fig. 2). The force required 


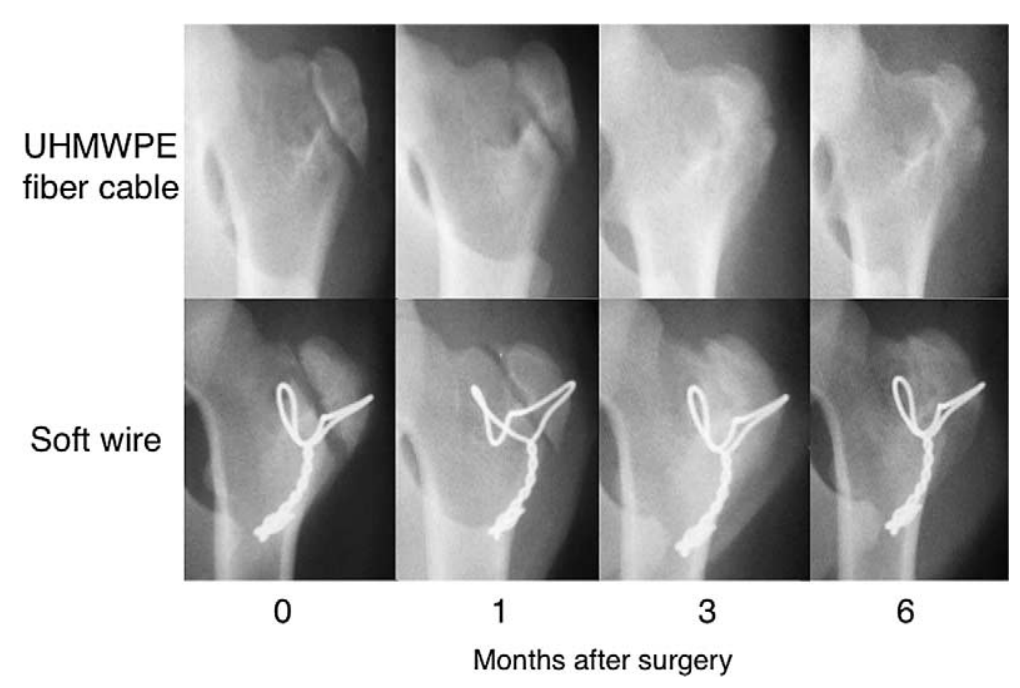

Fig. 3. Representative radiographs of the osteotomized greater trochanter. In both groups, the osteotomy site was visible until 1 month after surgery, but bone union was achieved by 3 months.

to remove the wire cable was significantly greater than that required for the UHMWPE fiber cable $(p<0.001)$. There was no significant difference between the UHMWPE fiber cable and soft wire $(p=0.219)$.

\subsection{Experiment 2}

The UHMWPE fiber cable showed similar union rates as the soft wire, at 88 and $100 \%$ at 3 and 6 months after operation, respectively, in both groups (Fig. 3). Bone union was also confirmed histologically in the osteosynthesis area of the sacrificed greater trochanter. In all samples in both groups, either callus union or trabecular bone formation was observed in the area 3 months after operation. At 6 months after surgery, complete bone union was observed in both groups.

In the surface area of the greater trochanter under the biomaterial, no significant tissue reaction was observed either in the soft wire group or in the UHMWPE fiber cable group (Fig. 4). Tissue reactions, including inflammatory reactions and granulation tissue formation, tended to be greater in the soft wire group than to the UHMWPE fiber cable group (Table 1). In the UHMWPE fiber cable group, a few foreign body giant cells could be seen adjacent to the cable.

In the penetration area of the greater trochanter in the soft wire group, there was a space between the wire and the surrounding bone tissue (Fig. 5). An inflammatory reaction was observed one month after the operation, and the space was later filled with granulation or fibrous tissue. In contrast, there was no space between the cable and the surrounding bone in the UHMWPE fiber cable group. No tissue invasion into the cable was observed. Foreign body giant cells were occasionally observed adjacent to the cable. There was a tendency toward an increased number of giant cells in the UHMWPE fiber cable group compared to the soft wire group (Table 2).

\section{Discussion}

In this study, a UHMWPE fiber cable was evaluated as a fixation biomaterial for osteosynthesis, and the clinical performances of this cable was compared with those of a soft wire and a wire cable. Each 

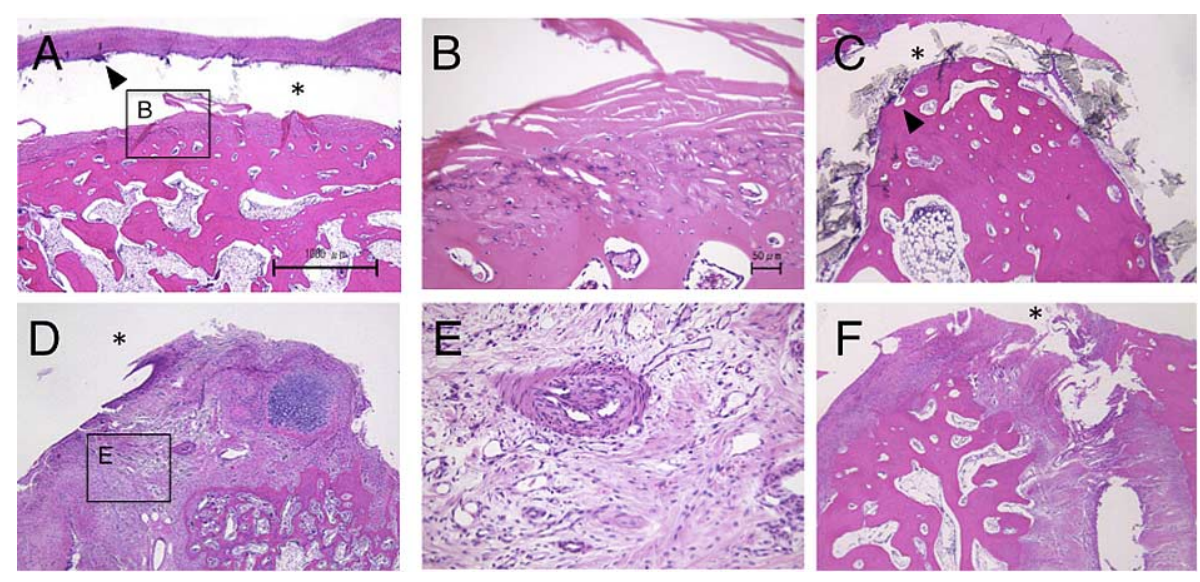

Fig. 4. Histology of the surface of the greater trochanter under the fixation material. (A), (B), (C): Representative samples of the UHMWPE fiber cable group. (D), (E), (F): Representative samples of the soft wire group. (A) and (D) show the area 1 month after surgery, and (B) and (E) are magnified views of the square indicated in (A) or (D), respectively. (C) and (F) show the area at 6 months. In the UHMWPE fiber cable group, no inflammatory reactions were observed. However, a few foreign body giant cells (arrow heads) were seen around the cable. The histological scores of (A) and (C) were 1 and 2 points with regard to the foreign body giant cells, respectively. In contrast, in the soft wire group, an inflammatory reaction was observed 1 month after surgery, and granulation tissue followed by fibrosis was subsequently formed adjacent to the wire. The score of the inflammatory reaction in (D) was 2, and the fibrosis score of (F) was 2. (Colors are visible in the online version of the article; http://dx.doi.org/10.3233/BME-130757.)

Table 1

Histological scores in the surface area of the greater trochanter

\begin{tabular}{|c|c|c|c|c|c|c|}
\hline & \multicolumn{2}{|c|}{ One month } & \multicolumn{2}{|c|}{3 months } & \multicolumn{2}{|c|}{6 months } \\
\hline & $\begin{array}{l}\text { UHMWPE } \\
\text { fiber cable }\end{array}$ & $\begin{array}{l}\text { Soft } \\
\text { wire }\end{array}$ & $\begin{array}{l}\text { UHMWPE } \\
\text { fiber cable }\end{array}$ & $\begin{array}{l}\text { Soft } \\
\text { wire }\end{array}$ & $\begin{array}{l}\text { UHMWPE } \\
\text { fiber cable }\end{array}$ & $\begin{array}{l}\text { Soft } \\
\text { wire }\end{array}$ \\
\hline Sample number & 4 & 4 & 4 & 4 & 4 & 4 \\
\hline \multicolumn{7}{|l|}{ Histological observation } \\
\hline Inflammatory reaction & 0.5 & 1 & 0 & 0.5 & 0 & 0 \\
\hline Granulation formation & 0.25 & 1 & 0 & 1 & 0 & 0 \\
\hline Fibrosis & 0 & 0 & 1.25 & 0.5 & 0.25 & 1.5 \\
\hline Foreign body giant cells & 1 & 0 & 1.5 & 0 & 1.75 & 0 \\
\hline
\end{tabular}

tightening method was standardized in order to minimize the surgeon's bias and was performed using identical methods with clinical touch. In the pull-out test used in Experiment 1, the removability of each fixation biomaterial was investigated after the material had been implanted in the bone tissue for 6 months. The UHMWPE fiber cable could be removed as easily as the soft wire, and both the UHMWPE fiber cable and the soft wire were significantly easier to remove than the wire cable. This difference in removability seemed to be due to differences in surface structures or biological reactivities. The surface of the soft wire was smooth, while the surface of the wire cable was relatively rough due to the bundle structure of many small fibers. In Experiment 2, histological analysis of the penetration area of the bone tissue showed that no invasion of the surrounding tissue was observed in the UHMWPE fiber cable group although this material has a bundle structure similar to the wire cable. The difference in removability between the UHMWPE fiber cable and the wire cable was probably due to differences in the biological reactivity of each biomaterial, i.e., polyethylene or titanium. 

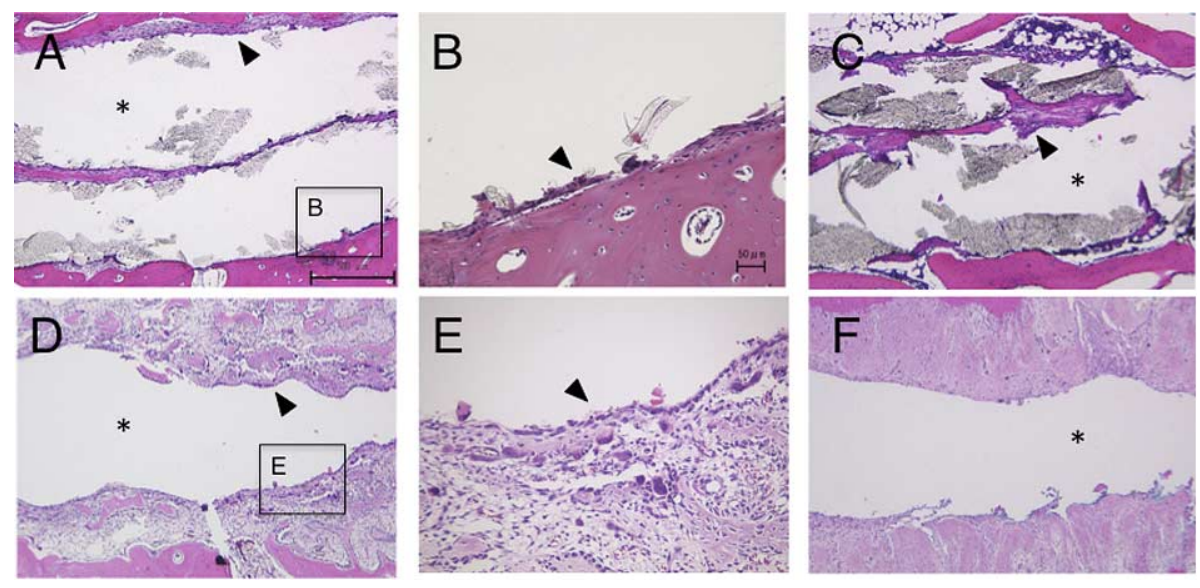

Fig. 5. Histology of the penetration area of the proximal femur around the fixation material. (A), (B), (C): Representative samples of the UHMWPE fiber cable group. (D), (E), (F): Representative samples of the soft wire group. (A) and (D) show the area at 1 month after surgery, and (B) and (E) are magnified views of the squares indicated in (A) and (D), respectively. (C) and (F) were taken at 6 months. In the UHMWPE fiber cable group, a slight inflammatory reaction was evident around the cable 1 month after surgery, and there were a few foreign body giant cells that persisted up to 6 months after surgery. The histological score of the inflammatory reaction in (A) was 1, and the scores of the foreign body giant cells were 1 at both 1 and 6 months after surgery. In contrast, in the soft wire group, an inflammatory reaction was observed around the wire 1 month after surgery, and fibrotic granulation tissue filled the area between the wire and the surrounding bone. The histological score of (D) was 2 for the inflammatory reaction, and the score of $(\mathrm{F})$ for fibrosis was 2. (Colors are visible in the online version of the article; http://dx.doi.org/10.3233/BME-130757.)

Table 2

Histological scores in the penetration area of the proximal femur

\begin{tabular}{|c|c|c|c|c|c|c|}
\hline & \multicolumn{2}{|c|}{ One month } & \multicolumn{2}{|c|}{3 months } & \multicolumn{2}{|c|}{6 months } \\
\hline & $\begin{array}{l}\text { UHMWPE } \\
\text { fiber cable }\end{array}$ & $\begin{array}{l}\text { Soft } \\
\text { wire }\end{array}$ & $\begin{array}{l}\text { UHMWPE } \\
\text { fiber cable }\end{array}$ & $\begin{array}{l}\text { Soft } \\
\text { wire }\end{array}$ & $\begin{array}{l}\text { UHMWPE } \\
\text { fiber cable }\end{array}$ & $\begin{array}{l}\text { Soft } \\
\text { wire }\end{array}$ \\
\hline Sample number & 4 & 4 & 4 & 4 & 4 & 4 \\
\hline \multicolumn{7}{|l|}{ Histological observation } \\
\hline Inflammatory reaction & 0.25 & 1.5 & 0 & 0.5 & 0 & 0 \\
\hline Granulation formation & 0.75 & 0.5 & 0 & 0.25 & 0 & 0 \\
\hline Fibrosis & 0 & 0 & 0.75 & 0 & 0 & 1.5 \\
\hline Foreign body giant cells & 1 & 0.5 & 1.5 & 0 & 1 & 0 \\
\hline
\end{tabular}

The fixation capability of the UHMWPE fiber cable was examined in an osteosynthesis model of the dog greater trochanter and was compared with that of the soft wire. Bone union appeared similar between the UHMWPE fiber cable and soft wire groups, both radiographically and histologically. As described in the Methods section, $25 \%$ of the animals in the soft wire group were excluded due to problems of fixation. In contrast, no animals were excluded in the UHMWPE fiber cable group, indicating that fixation of the osteotomized greater trochanter using the UHMWPE fiber cable was more stable than that using soft wire.

The biological reactivity of the UHMWPE fiber cable was investigated histologically in the osteosynthesis model and was compared with that of the soft wire. Both in the surface area of the greater trochanter under the fixation material and in the penetration area around the fixation material, a tendency toward an increased biological reactivity, including inflammatory reactions and granulation tissue 
formation, was observed in the soft wire group, as compared to the UHMWPE fiber cable group. Even in samples 1 month after the operation, there was no space between the UHMWPE fiber cable and the surrounding bone tissue in the penetration area. Since the hole in the bone was drilled large enough for the cable to pass through, there was some space between the cable and the surrounding bone tissue just after the operation. This indicates that new bone formation occurred between the UHMWPE fiber cable and the surrounding bone tissue after implantation. Additionally, no bone invasion into the cable was observed. These findings indicated that the UHMWPE cable did not interfere with new bone formation around the cable, which occurred naturally following the injury caused by the bone drilling. Biological reactions such as inflammatory reactions and granulation tissue formation around the soft wire may interfere with surrounding bone formation after the operation. The differences in biological reactivity between these 2 biomaterials may depend on the intrinsic properties of the biomaterial, such as mechanical properties, or fixation conditions.

This study has several limitations. The fixation capability and biological compatibility of the wire cable, which is sometimes used clinically to fix the osteotomized greater trochanter, were not compared with those of the UHMWPE cable since the wire cable was too bulky for use in the animal model chosen. According to the preliminary data described in the Methods section, the UHMWPE fiber cable had a withstand load and practical strength comparable to those of the wire cable. In a previous report concerning the spinal fixation system [11], a polyethylene cable was described as having a high strength capability for fixation, similar to a titanium or steel wire cable. Another limitation was that this study did not analyze the long-term effects of the UHMWPE cable on the surrounding bone, such as would be required when using the UHMWPE cable in arthroplasty. The histology around the UHMWPE cable occasionally showed few foreign body giant cells, but no bone resorption of the adjacent bone tissue was seen. There is a slight possibility that the UHMWPE cable could be a source of the polyethylene debris, which is known to induce osteolysis.

The UHMWPE fiber cable was as easily removable as the soft wire after being implanted in bone tissue and compared with a wire cable. Additionally, the UHMWPE fiber cable caused low biological reactivity with the surrounding tissue as compared with the soft wire and did not seem to interfere with bone formation. According to its clinical performance in spinal surgeries, the UHMWPE fiber cable should have good applicability for fixation in osteosynthesis. In conclusion, the UHMWPE fiber cable is a suitable fixation material for use in osteosynthesis.

\section{Acknowledgements}

This study was supported by a grant from Alfresa Pharma Co. The funds were used to pay for all aspects of the study, including the animals, chemicals and supplies. We would like to thank Yasumi Ishida, Tsutomu Morihara and Yasuo Sasai. We are also obliged to Dai Watanabe, Yoshikazu Nagashima, Makoto Ishigami, Kayoko Kudo and Koichi Suwa for their excellent technical assistance with this project.

\section{References}

[1] H.C. Amstutz and S. Maki, Complications of trochanteric osteotomy in total hip replacement, J. Bone Joint Surg. Am. 60 (1978), 214-216.

[2] N.S. Eftekhar, Total Hip Arthroplasty, CV Mosby, St. Louis, MO, 1993, pp. 16-61. 
[3] N.F. Jensen and W.H. Harris, A system for trochanteric osteotomy and reattachment for total hip arthroplasty with a ninety-nine percent union rate, Clin. Orthop. Relat. Res. 208 (1986), 174-181.

[4] R.W. Nutton and R.G. Checketts, The effects of trochanteric osteotomy on abductor power, J. Bone Joint Surg. Br. 66 (1984), 180-183.

[5] B.M. Wroblewski and P. Shelley, Reattachment of the greater trochanter after hip replacement, J. Bone Joint Surg. Br. 67 (1985), 736-740.

[6] S.S. Kelley and R.C. Johnston, Debris from cobalt-chrome cable may cause acetabular loosening, Clin. Orthop. Relat. Res. 285 (1992), 140-146.

[7] C.D. Silverton, J.J. Jacobs, A.G. Rosenberg et al., Complications of a cable grip system, J Arthroplasty 11 (1996), 400404.

[8] D.M. Dall and A.W. Miles, Re-attachment of the greater trochanter. The use of the trochanter cable-grip system, J. Bone Joint Surg. Br. 65 (1983), 55-59.

[9] M.A. Ritter, L.E. Eizember, E.M. Keating et al., Trochanteric fixation by cable grip in hip replacement, J. Bone Joint Surg. Br. 73 (1991), 580-581.

[10] I. Oh, T.W. Sander and R.W. Treharne, The fatigue resistance of orthopaedic wire, Clin. Orthop. Relat. Res. 192 (1985), $228-236$.

[11] C.A. Dickman, S.M. Papadopoulos, N.R. Crawford et al., Comparative mechanical properties of spinal cable and wire fixation systems, Spine 22 (1997), 596-604.

[12] H. Murakami, K. Yamazaki, E.S. Attallah-Wasif et al., A biomechanical study of 3 different types of sublaminar wire used for constructs in the thoracic spine, J. Spinal Disord. Tech. 19 (2006), 442-446.

[13] C.K. Naughton, J. Myles, A.J. Thomas Jr., The use of URYX for reversible vasectomy in a rabbit model, J. Androl. 25 (2004), 545-553. 